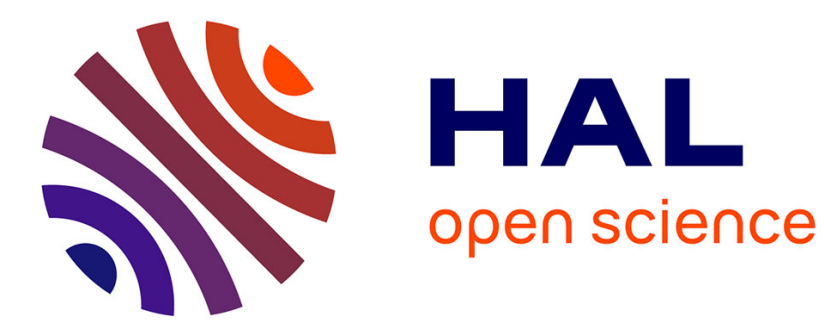

\title{
Full-waveform redatuming via a TRAC approach: a first step towards target oriented inverse problem
}

\author{
Franck Assous, Frédéric Nataf
}

\section{To cite this version:}

Franck Assous, Frédéric Nataf. Full-waveform redatuming via a TRAC approach: a first step towards target oriented inverse problem. Journal of Computational Physics, 2021, 440. hal-02530264v2

\section{HAL Id: hal-02530264 \\ https://hal.science/hal-02530264v2}

Submitted on 2 Jan 2021

HAL is a multi-disciplinary open access archive for the deposit and dissemination of scientific research documents, whether they are published or not. The documents may come from teaching and research institutions in France or abroad, or from public or private research centers.
L'archive ouverte pluridisciplinaire HAL, est destinée au dépôt et à la diffusion de documents scientifiques de niveau recherche, publiés ou non, émanant des établissements d'enseignement et de recherche français ou étrangers, des laboratoires publics ou privés. 


\title{
Full-waveform redatuming via a TRAC approach: a first step towards target oriented inverse problem
}

\author{
F. Assous ${ }^{1}$ and F. Nataf ${ }^{2}$ \\ ${ }^{1}$ Ariel University, Israël \\ ${ }^{2}$ Sorbonne Université, Paris, France
}

\section{Contents}

\begin{tabular}{lll}
\hline 1 & Introduction & 2
\end{tabular}

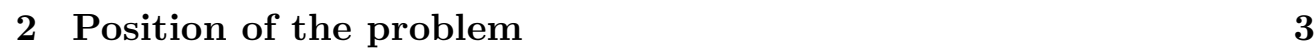

\begin{tabular}{|lll}
\hline 3 & Some redatuming methods & 4
\end{tabular}

3.1 TRAC method $\ldots \ldots \ldots \ldots \ldots \ldots \ldots$

3.2 Least Square with Neumann control . . . . . . . . . . . . . . 6

\begin{tabular}{|lll}
\hline 4 & Redatuming with TRAC-ls & 6
\end{tabular}

4.1 Adjoint State problem . . . . . . . . . . . . . . . . . . . 7

4.2 Computation of the gradient . . . . . . . . . . . . . . . 10

$\begin{array}{llr}5 & \text { Numerical Results } & 10\end{array}$

5.1 Problem setting . . . . . . . . . . . . . . . . . . . . . 11

5.2 Results without noise . . . . . . . . . . . . . . . . . 11

5.3 Results with noise $\ldots \ldots \ldots \ldots$. . . . . . . . . . . . . 15

5.4 Many Layers $\ldots \ldots \ldots \ldots$

\begin{tabular}{llr}
\hline 6 & Conclusion & 19
\end{tabular}

\begin{abstract}
In inverse problems, redatuming data consists in virtually moving the sensors from the original acquisition location to an arbitrary position. This is an essential tool for target oriented inversion. An exact redatuming method which has the peculiarity to be robust with respect to noise is proposed. Our iterative method is based on the Time Reversal Absorbing Conditions (TRAC) approach [1] and avoids the need for a regularization strategy. Numerical results and comparisons with other redatuming approaches illustrate the robustness of our method.
\end{abstract}




\section{Introduction}

Inverse problems play a crucial role in imaging whether in the medical field or in seismic exploration, and remains an active subject of research, with many applications such as tumor detection or stroke prevention in the case of medical imaging [16, 15]. In geophysics, seismic imaging is used as a tool for exploring subsoil for oil, gas or other deposits [23, 8]. From the mathematical point of view, parameter or signal estimation can be written as a partial differential equation constrained optimization problem [22, 12, 14], which tries to minimize the misfit between the recorded data and the reconstruction obtained by an estimated parameter or signal.

At each step of the optimization process, this estimation is updated to get closer to the original parameter to recover. This requires to solve at each iteration the so-called forward problem on the whole domain. The large size of the domain and the number of solves make this operation time-consuming and resource-intensive. Hence, redatuming, a target oriented approach which consists in reducing the inverse problem to a smaller region of interest, is very appealing. Redatuming data consists in virtually moving the sources and receivers from the original acquisition level to a new location. This method can be employed when the original acquisition location is inadequate for imaging purposes e.g. remoteness from target, irregular spatial sampling, etc. Redatuming relies on some prior knowledge of the medium parameters between the original and the new location which will be called overburden. The standard approach consists in modeling the propagation of the direct waves between the surface and the positions of the virtual sources/receivers that requires a velocity model of the overburden as prior information, see [5, 21]. These approaches usually are based on simplifying assumptions such as one-way wave propagation. The standard model-based redatuming methods can successfully focus the direct waves at the new location but do not account for surface and internal multiple reflections in the overburden. These multiples can generate ghost arrivals in the redatumed data set and thus ghost reflectors in the subsequent images. In more recent papers (e.g. [17] and [20] for a review), redatuming is formulated for the two-way wave equation. This means that events such as multiples and refractions are included. This is made possible by formulating redatuming as an inverse problem for the full acoustic wave equation. In [19] the Marchenko equations, see also [24] and references therein, are combined with a one-way version of the Rayleigh integral representation to obtain a new redatuming scheme that handles internal as well as free-surface multiples. Our approach is different and based on the full wave equation.

Inverse problems are in general ill-posed and need some regularization. We propose here an exact redatuming method which is robust with respect to noise in the data and avoids thus the not so obvious choice of a regularization function. Our method is based on the TRAC (Time Reversal Absorbing Conditions, see [1, 11]) method which is a blend of time reversal techniques [9] and absorbing boundary conditions [7, 3]. In its original version, it addressed the redatuming issue for homogeneous infinite domains with inclusions and was used in [6] to solve target oriented inverse problems. Here we introduce its generalization 


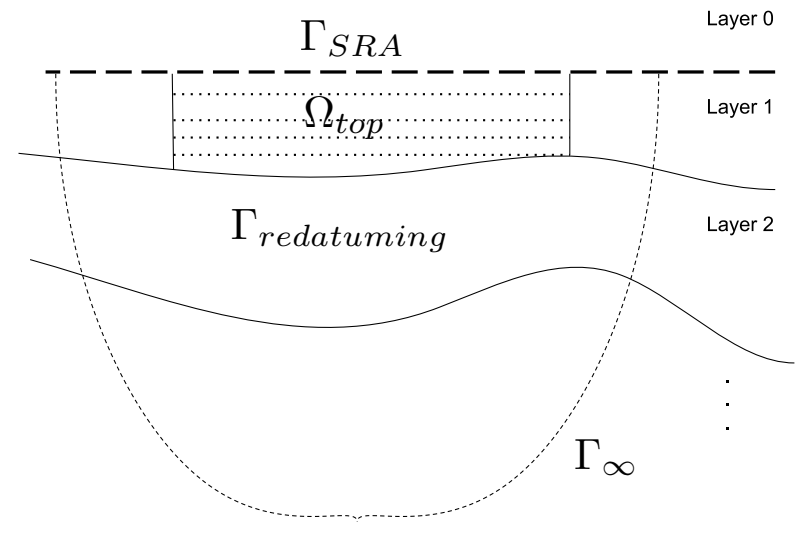

Figure 1: Stratified domain $\Omega$ with receivers on the top and the redatuming boundary $\Gamma_{\text {redatuming }}$

to inhomogeneous media e.g. a stratified domain made of layers which creates surface and internal multiple reflections. More precisely, in $\S 2$ we introduce the model redatuming problem, then in $\S 3$ we describe some existing approaches. The novel iterative method is derived in $\S 4$ along with the computation of the gradient by the adjoint state method. Numerical results that illustrate its robustness w.r.t. noise are shown in $\S 5$. Comparisons with the methods mentioned in $\S 3$ are also performed.

\section{Position of the problem}

Let $\Omega$ be a domain made of several layers numbered $1,2, \ldots$ with a boundary $\Gamma_{S R A}$ (SRA stands for Source-Receivers Array) on the top and a truncation boundary $\Gamma_{\infty}$ where we impose an absorbing boundary condition, see Fig. 1 . Let $f(t, x)$ be a given source term located at $x_{S} \in \Omega$ :

$$
f(t, x):=\operatorname{Ricker}(t) \times \delta_{x=x_{S}},
$$

where the Ricker function is of the form for $t<T_{S}$ :

$$
\left(1-2 \pi^{2}\left(\nu_{0} t-1\right)^{2}\right) \exp \left(-\pi^{2}\left(\nu_{0} t-1\right)^{2}\right) \times 1_{\left[0, T_{S}\right]} .
$$

Above, $\nu_{0}$ is the central frequency and $T_{S}$ is the emission time of the source. Let us denote by $c(x)$ the wave velocity and by $n$ the outward unit normal on the boundary. We assume in addition that Layer 0 is made of air so that we can impose a free surface boundary condition on the boundary $\Gamma_{S R A}$. We define 
the forward problem that computes the (total) pressure $p^{T}(t, x)$ as follows:

$$
\begin{aligned}
\frac{1}{c^{2}(x)} \frac{\partial^{2} p^{T}}{\partial t^{2}}-\Delta p^{T} & =f(t, x) \text { in } \Omega \\
\frac{\partial p^{T}}{\partial t}+c(x) \frac{\partial p^{T}}{\partial n} & =0 \text { on } \Gamma_{\infty} \\
p^{T} & =0 \text { on } \Gamma_{S R A} \\
p^{T}(0, x) & =0 \text { and } \frac{\partial p^{T}}{\partial t}(0, x)=0 \quad \forall x \in \Omega .
\end{aligned}
$$

Assuming the receivers are located on the free surface boundary $\Gamma_{S R A}$, we record, on $\Gamma_{S R A}, u_{o b s}:=\partial p^{T} / \partial n$ the normal derivative of the pressure which corresponds to the time derivative of the normal velocity up to a multiplicative constant. We introduce a subdomain $\Omega_{t o p}$ (see Fig. 1) which is part of Layer 1 whose physical properties are assumed to be known. Notice that we will reconstruct the signal in the whole domain $\Omega_{\text {top }}$. Let $\Gamma_{\text {redatuming }}:=\partial \Omega_{t o p} \backslash \Gamma_{S R A}$ be a part of the boundary of the domain where we aim at redatuming the recorded data $u_{o b s}$. We assume that after some time $T_{f}$, the total pressure $p^{T}$ vanishes in the domain $\Omega_{t o p}$.

\section{Some redatuming methods}

From the recorded field $u_{o b s}$ and assuming the knowledge of the velocity field $c$ in $\Omega_{\text {top }}$, our aim is to reconstruct $p(t, x)$ on $\left(0, T_{f}\right) \times \Gamma_{\text {redatuming }}$. We detail two methods that are the origin of the new method we propose in section 4. As a first step, we compute an incident pressure in the domain $\Omega$ solution to:

$$
\begin{aligned}
\frac{1}{c_{I}^{2}} \frac{\partial^{2} p^{I}}{\partial t^{2}}-\Delta p^{I} & =f(t, x) \text { in }\left(0, T_{f}\right) \times \Omega \\
\frac{\partial p^{I}}{\partial t}+c_{I} \frac{\partial p^{I}}{\partial n} & =0 \text { on }\left(0, T_{f}\right) \times \Gamma_{\infty} \\
p^{I} & =0 \text { on } \Gamma_{S R A} \\
p^{I}(0, x) & =0 \text { and } \frac{\partial p^{I}}{\partial t}(0, x)=0, \quad \forall x \in \Omega,
\end{aligned}
$$

where $c_{I}$ is the value of the velocity in the first layer (Layer 1) which is assumed to be known. Let $u_{r e f}^{S}(t, x):=u_{o b s}(t, x)-\frac{\partial p^{I}}{\partial n}$ for $x \in \Gamma_{S R A}$. The scattered pressure $p^{S}(t, x):=p^{T}(t, x)-p^{I}(t, x)$ satisfies in $\Omega_{t o p}$ :

$$
\begin{aligned}
\frac{1}{c_{I}^{2}} \frac{\partial^{2} p^{S}}{\partial t^{2}}-\Delta p^{S} & =0 \text { in }\left(0, T_{f}\right) \times \Omega_{t o p} \\
p^{S}=0 \text { and } \frac{\partial p^{S}}{\partial n} & =u_{r e f}^{S}(t, x) \text { on }\left(0, T_{f}\right) \times \Gamma_{S R A} \\
p^{S}(0, x) & =0 \text { and } \frac{\partial p^{S}}{\partial t}(0, x)=0, \quad \forall x \in \Omega_{t o p} .
\end{aligned}
$$


The boundary value problem (4) is overdetermined on the free surface boundary $\Gamma_{S R A}$ due to the knowledge of both the pressure and its normal derivative whereas it is underdetermined on the redatuming boundary $\Gamma_{\text {redatuming }}$ where it is unknown. In subsections 3.1 and 3.2 , we briefly recall two redatuming methods to solve the inverse problem consisting of reconstructing $p^{S}$ on $\Gamma_{\text {redatuming. }}$.

\subsection{TRAC method}

In previous papers [1, 2], we introduced a method that enables one to "recreate the past" of a wave scattered by unknown scatterers provided that the surrounding domain is infinite and homogeneous. This was made possible by blending time reversal techniques and absorbing boundary conditions so that the method was named TRAC (Time Reversed Absorbing Condition). In the case where Layer 0 and Layer 1 have the same physical properties with the same constant velocity, the boundary condition $p=0$ on the SRA boundary makes no sense and the TRAC method solves our problem. Let us describe this approach now.

In this time reversal technique, we compute the time reversed pressure $q_{R}^{S}(t, x):=p^{S}\left(T_{f}-t, x\right)$ by solving:

$$
\begin{array}{r}
\frac{1}{c^{2}} \frac{\partial^{2} q_{R}^{S}}{\partial t^{2}}-\Delta q_{R}^{S}=0 \text { in }\left(0, T_{f}\right) \times \Omega_{t o p} \\
\frac{\partial q_{R}^{S}}{\partial n}=u_{r e f}^{S}\left(T_{f}-t, x\right) \text { on }\left(0, T_{f}\right) \times \Gamma_{S R A} \\
T R A C\left(q_{R}^{S}\right)=0 \text { on } \Gamma_{\text {redatuming }} \\
q_{R}^{S}(0, x)=0 \text { and } \frac{\partial q_{R}^{S}}{\partial t}(0, x)=0, \quad \forall x \in \Omega_{t o p} .
\end{array}
$$

In the system above, eq. (5) is justified by the time reversibility of the wave equation and eq. (6) expresses that the data are recorded on $\Gamma_{S R A}$. The last equation (8) reflects the fact that after time $T_{f}$ the scattered pressure vanishes. Had Layer 0 and Layer 1 had the same constant physical properties, the pressure $q_{R}^{S}$ would be an outgoing field in Layers 0 and 1 that would satisfy eq. (7) where $T R A C$ denotes the time reversal of an absorbing boundary condition (ABC). For illustration in two dimensions, if a valid ABC is the Bayliss-Turkel condition

$$
B T 1=\frac{\partial}{\partial t}+c \frac{\partial}{\partial n}+\frac{c}{2 r}
$$

then, $T R A C$ reads in this case:

$$
T R A C:=\frac{\partial}{\partial t}+c \frac{\partial}{\partial n}-\frac{c}{2 r} .
$$

For a detailed derivation, see [1].

Remark 1 One-way wave equation enables to compute an approximation to the downgoing part of the scattered pressure, [5, 4]. This technique is cheap 
but the computed pressure does not satisfy the wave equation in all of $\Omega_{\text {top }}$. At a higher cost, the TRAC method also computes the downgoing part but which satisfies the wave equation in all of $\Omega_{\text {top }}$ and thus extends the approximation's range of validity of the redatuming approach.

In the present case, Layer 0 and Layer 1 do not have the same physical properties and eq. (7) is not satisfied so that the corresponding redatuming method denoted by TRAC is not accurate, see 5 .

\subsection{Least Square with Neumann control}

We now turn to an approach related to the classical framework of inverse problems. A standard formulation is to consider the value of the normal derivative of the time reversed pressure $\frac{\partial p_{R}^{S}(t, x)}{\partial n}:=\frac{\left.\partial p^{S}\left(T_{f}-t, x\right)\right)}{\partial n}$ on $\Gamma_{\text {redatuming }}$ as the unknown to be reconstructed. To this end, we look for $g:\left(0, T_{f}\right) \times \Gamma_{\text {redatuming }} \rightarrow$ $\mathbf{R}$ such that the time reversed pressure $q_{R}^{S}(t, x)$ solution to the following BVP

$$
\begin{array}{r}
\frac{1}{c^{2}} \frac{\partial^{2} q_{R}^{S}}{\partial t^{2}}-\Delta q_{R}^{S}=0 \text { in }\left(0, T_{f}\right) \times \Omega_{\text {top }} \\
\frac{\partial q_{R}^{S}}{\partial n}=u_{r e f}^{S}\left(T_{f}-t, x\right) \text { on }\left(0, T_{f}\right) \times \Gamma_{S R A} \\
\frac{\partial q_{R}^{S}}{\partial n}=g \text { on }\left(0, T_{f}\right) \times \Gamma_{\text {redatuming }} \\
q_{R}^{S}(0, x)=0 \text { and } \frac{\partial q_{R}^{S}}{\partial t}(0, x)=0, \quad \forall x \in \Omega_{\text {top }},
\end{array}
$$

satisfies $q_{R}^{S}$ equals zero on the top boundary $\Gamma_{S R A}$ for all time $t \in\left(0, T_{f}\right)$. In the sequel, this method will be referred to as Neumann-ls.

In order to determine function $g$ in an iterative procedure, let us introduce a cost function $J_{N}(g)$ defined by:

$$
J_{N}(g):=\frac{1}{2} \int_{0}^{T_{f}} \int_{\Gamma_{S R A}}\left|q_{R}^{S}\right|^{2}+\frac{\alpha}{2} \int_{0}^{T_{f}} \int_{\Gamma_{\text {redatuming }}}|g(t, s)|^{2} d s,
$$

where $\alpha \geq 0$ is a regularization parameter and $q_{R}^{S}$ is solution to $10-113$. We use the conjugate gradient method to minimize $J_{N}$. Once $g$ has been found, we obtain $q_{R}^{S}(t, x)$ by solving (10)-13) and the pressure $p^{S}(t, x)$ is actually the time reversed of $q_{R}^{S}(t, x)$ i.e. $p^{S}(t, x)=q_{R}^{S}\left(T_{f}-t, x\right)$.

In contrast to the previous method $\S 3.1$, note that even when physical properties of Layers 0 and 1 are the same the Neumann-ls method yields the solution only after an iterative process.

\section{Redatuming with TRAC-ls}

Our idea is to use the best of both worlds by combining the advantage of each method mainly by deriving an iterative TRAC method that will be named 
TRAC-ls where ls stands for least square. For the sake of completness, we detail the equations below. We look for $g:\left(0, T_{f}\right) \times \Gamma_{\text {redatuming }} \rightarrow \mathbf{R}$ such that the time reversed pressure $q_{R}^{S}(t, x)$ solution to the following boundary value problem (BVP)

$$
\begin{array}{r}
\frac{1}{c^{2}} \frac{\partial^{2} q_{R}^{S}}{\partial t^{2}}-\Delta q_{R}^{S}=0 \text { in }\left(0, T_{f}\right) \times \Omega_{t o p} \\
\frac{\partial q_{R}^{S}}{\partial n}=u_{r e f}^{S}\left(T_{f}-t, x\right) \text { on } \Gamma_{S R A} \\
T R A C\left(q_{R}^{S}\right)=\left(\frac{\partial}{\partial t}+c \frac{\partial}{\partial n}-\frac{c}{2 r}\right)\left(q_{R}^{S}\right)=g \text { on } \Gamma_{\text {redatuming }} \\
q_{R}^{S}(0, x)=0 \text { and } \frac{\partial q_{R}^{S}}{\partial t}(0, x)=0, \quad \forall x \in \Omega_{t o p},
\end{array}
$$

is such that $q_{R}^{S}$ equals zero on the top boundary $\Gamma_{S R A}$ for all time $t \in\left(0, T_{f}\right)$. We assume that the inverse problem is well posed so that condition (17) ensures that $p^{S}(t, x)$ is the time reversed pressure of $q_{R}^{S}\left(T_{f}-t, x\right)$ i.e. the following equality holds $p^{S}(t, x)=q_{R}^{S}\left(T_{f}-t, x\right)$.

Remark 2 Note that the standard TRAC method $\$ 3.1$ can be viewed as a one shot method where equations (15)-(18) are solved with $g=0$.

Similarly to $\S 3.2$, we determine function $g$ by solving a least square problem. Let us introduce a cost function $J(g)$ defined by:

$$
J(g):=\frac{1}{2} \int_{0}^{T_{f}} \int_{\Gamma_{S R A}}\left|q_{R}^{S}\right|^{2}+\frac{\alpha}{2} \int_{0}^{T_{f}} \int_{\Gamma_{\text {redatuming }}}|g(t, s)|^{2} d s,
$$

where $\alpha \geq 0$ is a regularization parameter and $q_{R}^{S}$ is solution to (15)-(18). We use conjugate gradient method to minimize $J$. Clearly functions $J_{N}(g)$ and $J(g)$ are different due to the difference between equations (12) and (17).

\subsection{Adjoint State problem}

In order to minimize the cost function (19) by a conjugate gradient method, we compute its derivative by the well-known adjoint state method. For sake of completness, we detail this computation. For this purpose, we start from a variational formulation in space and time of (15)-18). Let $V$ denote

$$
V:=\left\{v \in H^{1}\left(\left(0, T_{f}\right) \times \Omega_{t o p}\right) \mid \text { and } v(0, \cdot)=\partial v / \partial t(0, \cdot)=0\right\} .
$$

We multiply equation (15) by a test function $v \in V$ and integrate by part in space:

$$
\int_{0}^{T_{f}} \int_{\Omega_{t o p}} \frac{1}{c^{2}} \frac{\partial^{2} q_{R}^{S}}{\partial t^{2}} v+\nabla q_{R}^{S} \cdot \nabla v-\int_{0}^{T_{f}} \int_{\partial \Omega_{t o p}} \frac{\partial q_{R}^{S}}{\partial n} v=0
$$


so that using (16) and (17), i.e. the Neumann boundary condition on $\Gamma_{S R A}$ and the TRAC boundary condition on $\Gamma_{\text {redatuming, }}$, we get:

$$
\begin{array}{r}
\int_{0}^{T_{f}} \int_{\Omega_{\text {top }}} \frac{1}{c^{2}} \frac{\partial^{2} q_{R}^{S}}{\partial t^{2}} v+\nabla q_{R}^{S} \cdot \nabla v+\int_{0}^{T_{f}} \int_{\Gamma_{\text {redatuming }}}\left(\frac{1}{c} \frac{\partial q_{R}^{S}}{\partial t}-\frac{1}{2 r} q_{R}^{S}\right) v \\
=\int_{0}^{T_{f}} \int_{\Gamma_{\text {redatuming }}} g v+\int_{0}^{T_{f}} \int_{\Gamma_{S R A}} u_{r e f}^{S}\left(T_{f}-t, x\right) v .
\end{array}
$$

We denote

$$
a(u, v):=\int_{0}^{T_{f}} \int_{\Omega_{\text {top }}}\left(\frac{1}{c^{2}} \frac{\partial^{2} u}{\partial t^{2}} v+\nabla u \cdot \nabla v\right)+\int_{0}^{T_{f}} \int_{\Gamma_{\text {redatuming }}}\left(\frac{1}{c} \frac{\partial u}{\partial t}-\frac{1}{2 r} u\right) v
$$

and

$$
l(v, g):=\int_{0}^{T_{f}} \int_{\Gamma_{\text {redatuming }}} g v+\int_{0}^{T_{f}} \int_{\Gamma_{S R A}} u_{r e f}^{S}\left(T_{f}-t, x\right) v .
$$

For a given $g$, we denote by $R(g)$ the unique function (i.e. $q_{R}^{S}$ ) in $V$ that satisfies

$$
a(R(g), v)=l(v, g), \forall v \in V,
$$

and by $\mathcal{L}$ the functional from $L^{2}\left(\left(0, T_{f}\right) \times \Gamma_{\text {redatuming }}\right) \times V \times V$ to $\mathbf{R}$ :

$$
\mathcal{L}(g, u, v):=\frac{1}{2} \int_{0}^{T_{f}} \int_{\Gamma_{S R A}}|u|^{2}+\frac{\alpha}{2} \int_{0}^{T_{f}} \int_{\Gamma_{\text {redatuming }}}|g|^{2}+a(u, v)-l(v, g) .
$$

Note that for arbitrary $v \in V$ we have the equality:

$$
J(g)=\mathcal{L}(g, R(g), v) .
$$

We differentiate the above equation w.r.t. $g$ and we get for all $v$ :

$$
<\nabla J(g), \delta g>=<\frac{\partial \mathcal{L}}{\partial g}(g, R(g), v), \delta g>+<\frac{\partial \mathcal{L}}{\partial u}(g, R(g), v), \delta u>
$$

where $\delta u \in V$ is defined by

$$
\delta u=<\nabla R(g), \delta g>
$$

We apply formula $(23)$ to the function $\mathcal{L}$ :

$$
\begin{aligned}
<\nabla J(g), \delta g>= & -\int_{0}^{T_{f}} \int_{\Gamma_{\text {redatuming }}} \delta g v+\alpha \int_{0}^{T_{f}} \int_{\Gamma_{\text {redatuming }}} g \delta g \\
& +\int_{0}^{T_{f}} \int_{\Gamma_{S R A}} q_{R}^{S} \delta u+a(\delta u, v)
\end{aligned}
$$

where we replaced $R(g)$ by $q_{R}^{S}$. In order to cancel the last term in eq. 24), we introduce $v^{*} \in V$ the adjoint state that satisfies for all $\delta u \in V$ :

$$
a\left(\delta u, v^{*}\right)=-\int_{0}^{T_{f}} \int_{\Gamma_{S R A}} q_{R}^{S} \delta u .
$$


Thus, equation 24 will no longer relies on $\delta u$ but only on $\delta g$. From the knowledge of the adjoint state $v^{*}$, we get:

$$
<\nabla J(g), \delta g>=-\int_{0}^{T_{f}} \int_{\Gamma_{\text {redatuming }}} \delta g v^{*}+\alpha \int_{0}^{T_{f}} \int_{\Gamma_{\text {redatuming }}} g \delta g d s
$$

so that we can identify

$$
\nabla J(g)=-v_{\mid \Gamma_{\text {redatuming }}}^{*}+\alpha g .
$$

From the weak formulation (25) of the the adjoint problem, we now identify the initial boundary value problem satisfied by $v^{*}$. For this purpose, we first reformulate $a$ by integrating by parts in time eq. 21):

$$
\begin{aligned}
a\left(\delta u, v^{*}\right) & =\int_{0}^{T_{f}} \int_{\Omega_{\text {top }}} \frac{1}{c^{2}} \delta u \frac{\partial^{2} v^{*}}{\partial t^{2}}-\left[\int_{\Omega_{\text {top }}} \frac{1}{c^{2}} \delta u \frac{\partial v^{*}}{\partial t}\right]_{0}^{T_{f}}+\left[\int_{\Omega_{\text {top }}} \frac{1}{c^{2}} \frac{\partial \delta u}{\partial t} v^{*}\right]_{0}^{T_{f}} \\
& +\int_{0}^{T_{f}} \int_{\Omega_{\text {top }}} \nabla \delta u \nabla v^{*}-\int_{0}^{T_{f}} \int_{\Gamma_{\text {redatuming }}} \frac{\delta u}{c} \frac{\partial v^{*}}{\partial t}+\left[\int_{\Gamma_{\text {redatuming }}} \frac{\delta u}{c} v^{*}\right]_{0}^{T_{f}} \\
& -\int_{0}^{T_{f}} \int_{\Gamma_{\text {redatuming }}} \frac{1}{2 r} \delta u v^{*} .
\end{aligned}
$$

Then integrating by part in space the above expression, plugging it in eq. 25) and finally identifying term by term, we see that $v^{*}$ satisfies the backpropagation problem:

$$
\begin{aligned}
\frac{1}{c^{2}} \frac{\partial^{2} v^{*}}{\partial t^{2}}-\Delta v^{*} & =0 \text { in }\left(0, T_{f}\right) \times \Omega_{\text {top }} \\
\frac{\partial v^{*}}{\partial t}-c \frac{\partial v^{*}}{\partial n}+\frac{c}{2 r} v^{*} & =0 \text { on }\left(0, T_{f}\right) \times \Gamma_{\text {redatuming }} \\
\frac{\partial v^{*}}{\partial n} & =-q_{R}^{S} \text { on }\left(0, T_{f}\right) \times \Gamma_{S R A} \\
v^{*}\left(T_{f}, x\right) & =0 \text { and } \frac{\partial v^{*}}{\partial t}\left(T_{f}, x\right)=0, \quad \forall x \in \Omega_{t o p} .
\end{aligned}
$$

Remark 3 Note that the boundary condition on $\Gamma_{\text {redatuming }}$ in (27) is not a BT1 absorbing boundary condition [3]. Indeed, problem [27) has final conditions at time $t=T_{f}$ so that it has to be solved as a reversed problem in time. Then, the boundary condition on the time reversed function $v_{R}^{*}(t, \cdot):=v^{*}\left(T_{f}-t, \cdot\right)$ on $\Gamma_{\text {redatuming }}$ reads after multiplication by -1 :

$$
\frac{\partial v_{R}^{*}}{\partial t}+c \frac{\partial v_{R}^{*}}{\partial n}-\frac{c}{2 r} v_{R}^{*}=0 \text { on }\left(0, T_{f}\right) \times \Gamma_{\text {redatuming }} .
$$

Due to the minus sign before the term $1 / 2 r$, this condition is not the $B T^{1}$ absorbing boundary condition. 


\subsection{Computation of the gradient}

In summary, for a given $g:\left(0, T_{f}\right) \times \Gamma_{\text {redatuming }} \mapsto \mathbf{R}$, the gradient $\nabla J(g)$ of the cost function $J$ at point $g$ is obtained via the following steps:

1. Compute $q_{R}^{S}$ solution of eq. (15)-18

2. Compute the adjoint state $v^{*}$ solution of 27)

3. The gradient $\nabla J(g):\left(0, T_{f}\right) \times \Gamma_{\text {redatuming }} \mapsto \mathbf{R}$ has the following expression: $\nabla J(g)=-v_{\mid \Gamma_{\text {redatuming }}^{*}}^{*}+\alpha g$, see eq. (26).

Remark 4 For the sake of completness, we have introduced a regularization parameter $\alpha \geq 0$ in the cost function $J$. However, all of our numerical experiments are performed without penalization, i.e. $\alpha=0$.

\section{$5 \quad$ Numerical Results}

In this section, our goal is to illustrate with numerical results the methods proposed above to retrieve the scattered pressure $p^{S}$ in domain $\Omega_{t o p}$ and particularly at the redatuming boundary $\Gamma_{\text {redatuming }}$, see Fig. 1 . The domain $\Omega_{\text {top }}$ is actually the domain between $\Gamma_{S R A}$ and $\Gamma_{\text {redatuming. }}$. We test mainly the three redatuming methods presented in $\S 3$. TRAC, Neumann-ls and TRAC-ls. The first method described in $\S 3.1$ consists in solving only one time reversed problem in $\Omega_{t o p}$. The second one is an iterative method where we seek the Neumann data on the boundary $\Gamma_{\text {redatuming }}$ such that the solution to $10-13$ vanishes on the boundary $\Gamma_{S R A}$. The third one is also iterative, but instead of looking for the Neumann data on $\Gamma_{\text {redatuming }}$ we rather search for the value of TRAC on $\Gamma_{\text {redatuming }}$, see eq. (17). In addition, it will be interesting in some situations to also consider a so-called "Dirichlet-ls" method in which we replace in (17) the TRAC operator by

$$
\beta \frac{\partial}{\partial t}+c \frac{\partial}{\partial n}-\beta \frac{c}{2 r},
$$

where $\beta$ is a real parameter equals to $10^{6}$. Operator $(28)$ allows us to play easily with the quantity which is optimized. Indeed taking $\beta=1$ corresponds to the TRAC-ls method whereas $\beta=10^{-20}$ is our way to implement the Neumann-ls method. Note also that the Dirichlet-ls method turns out to give bad results. For this reason, we will show its results only at the end of $\S 5.2$.

For all methods we first generate synthetic data by solving the forward problem (2) and the incident field by solving (3) on the whole domain $\Omega$. The scattered field $p^{S}$ is the difference between the forward field and the incident field. 


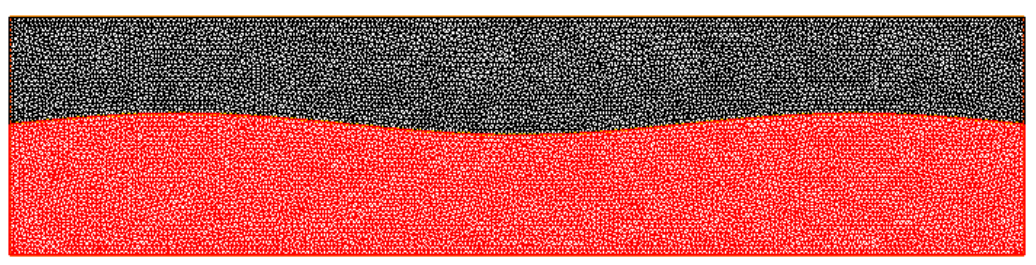

Figure 2: Two-layer domain $\Omega$ with SRA on the top boundary

\subsection{Problem setting}

We illustrate our method by comparing it with the approaches presented above in $\S 3.1$ and $\S 3.2$. We consider a configuration that mimics first a simple geophysics problem and then in $\S 5.4$ a more realistic geometry. Since our geometry is made of basically flat layers, in the TRAC eq. (9) the origin of the polar coordinates is located at a deep depth.

For the first tests, the domain $\Omega$ of length $3.3 \mathrm{~km}$ is made of two layers, see Fig. 2. Velocity is $c_{1}:=3200 \mathrm{~m} / \mathrm{s}$ in Layer 1 and $c_{2}:=2800 \mathrm{~m} / \mathrm{s}$ in Layer 2 . The width of the first layer is approximately $Z_{1}=450$ meters. The source as defined in eq. (1) is located in Layer 1 at a depth of $50 \mathrm{~m}$. Its central frequency is $\nu_{0}=15 \mathrm{~Hz}$ so that the horizontal length of the domain is approximately 20 times the mean wavelength. The emission time is $T_{S}:=2 / \nu_{0}$. The top boundary is the soil surface where we impose a pressure equal to zero. On the other boundaries we impose absorbing boundary conditions in the forward problem used to generate the recorded data on the surface. The total simulation time $T_{f}:=10 c_{1} / Z_{1}$ is taken so that the wave is nearly zero at the end of the computation. Note that $T_{f}$ corresponds to ten times the travel time from the surface to the interface between the layers. Thus, the wave undergoes five multiple reflections.

For numerical simulations, the wave equation (2) is approximated by the FreeFem package [13] which implements finite element methods in space. In this study we use a standard $P^{1}$ finite element method. The advancement in time is given by a second-order central finite difference scheme so that it is time reversible also on the numerical level. The incident wave is simulated by the same procedure with a uniform velocity of sound, $c_{1}$.

\subsection{Results without noise}

In order to better understand the propagation of the scattered field $p^{S}$, we start by presenting in Fig. 3 the snapshots of the reconstructed scattered signal. The first column corresponds to the exact solution, the second column to the TRACls method, the third one to Neumann-ls method and the last one to the TRAC method (i.e. without optimization process). On the first column, on the first row the scattered field $p^{S}$ is zero until it is partially reflected on the interface which corresponds to $\Gamma_{\text {redatuming }}$ (row 2). Note that the reconstruction takes place only in domain $\Omega_{t o p}$ so that the transmitted wave cannot be represented. Then the signal comes back to the boundary $\Gamma_{S R A}$ where it is totally reflected 
(rows $3-4$ ). We have then a multiple reflection process until the signal leaves the computational domain (three last rows).

In order to give a quantitative assessment of the different methods, we introduce first the global $L^{2}$ error defined by

$$
\int_{0}^{T_{f}} \int_{\Omega_{\text {top }}}\left(p_{\text {method }}^{S}-p_{\text {exact }}^{S}\right)^{2} d t d x
$$

where $p_{\text {exact }}^{S}$ (resp. $p_{\text {method }}^{S}$ ) is the exact (resp. reconstructed by one of the considered method) scattered field. We also compute for the same quantities the $L^{2}$ error on $\Gamma_{\text {redatuming }}$ defined by

$$
\int_{0}^{T_{f}} \int_{\Gamma_{\text {redatuming }}}\left(p_{\text {method }}^{S}-p_{\text {exact }}^{S}\right)^{2} d t d s .
$$

In Table 1, we give these two quantities in the first two lines for the three methods described above: Neumann-ls, TRAC and TRAC-ls. The third line gives the value of the cost function after 34 iterations for the iterative methods Neumann-ls and TRAC-ls. Recall that the TRAC method is actually the first iteration of the TRAC-ls method. The best results are given by the TRAC-ls method whereas the TRAC method behaves somehow better than the Neumann-ls method. For each method, we also display $p_{\text {method }}^{S}$ on the middle of the boundary $\Gamma_{\text {redatuming }}$ and give as reference the exact solution, see Fig. 4. Here again, the TRAC-ls method yields a good approximation of the exact solution. Contrarily, the Neumann-ls method provides a solution very different from the exact one, exhibiting large oscillations. Note that the TRAC method is able to recover the first arrival event but not the second one due to the reflection on $\Gamma_{S R A}$ where the pressure $p$ is equal to zero. This justifies the iterative procedure of the TRAC-ls method. In this condition, it is interesting to investigate the convergence of the TRAC-ls method. For this purpose, we show in Fig. 5 the reconstructed solution $p_{T R A C-l s}^{S}$ at the middle of the boundary $\Gamma_{\text {redatuming }}$ for different iterations of the conjugate gradient method.

\begin{tabular}{|c|c|c|c|c|}
\hline & Noise level & Neumann-ls & TRAC & TRAC-ls \\
\hline L2-error (global) & $0 \%$ & 93.1 & 92.9 & 38.5 \\
\hline L2-error (redatuming sensors) & $0 \%$ & 31.5 & 20.3 & 13.8 \\
\hline cost function & $0 \%$ & 0.0162 & 0.0117 & 0.0011 \\
\hline
\end{tabular}

Table 1: Errors and cost function value for the different reconstruction methods (without noise in the data)

In the same spirit, we consider now the Dirichlet-ls method. We first noticed that even without noise the conjugate gradient method suffers from a lack of convergence after iteration 13. This is not the case for the Neumann-ls and TRAC-ls methods, see Fig. 7. For this reason, except here we will not consider any longer the Dirichlet-ls method. In Fig. 6, we plot both the reconstructed scattered signals (top) and its normal derivative (bottom) on the 

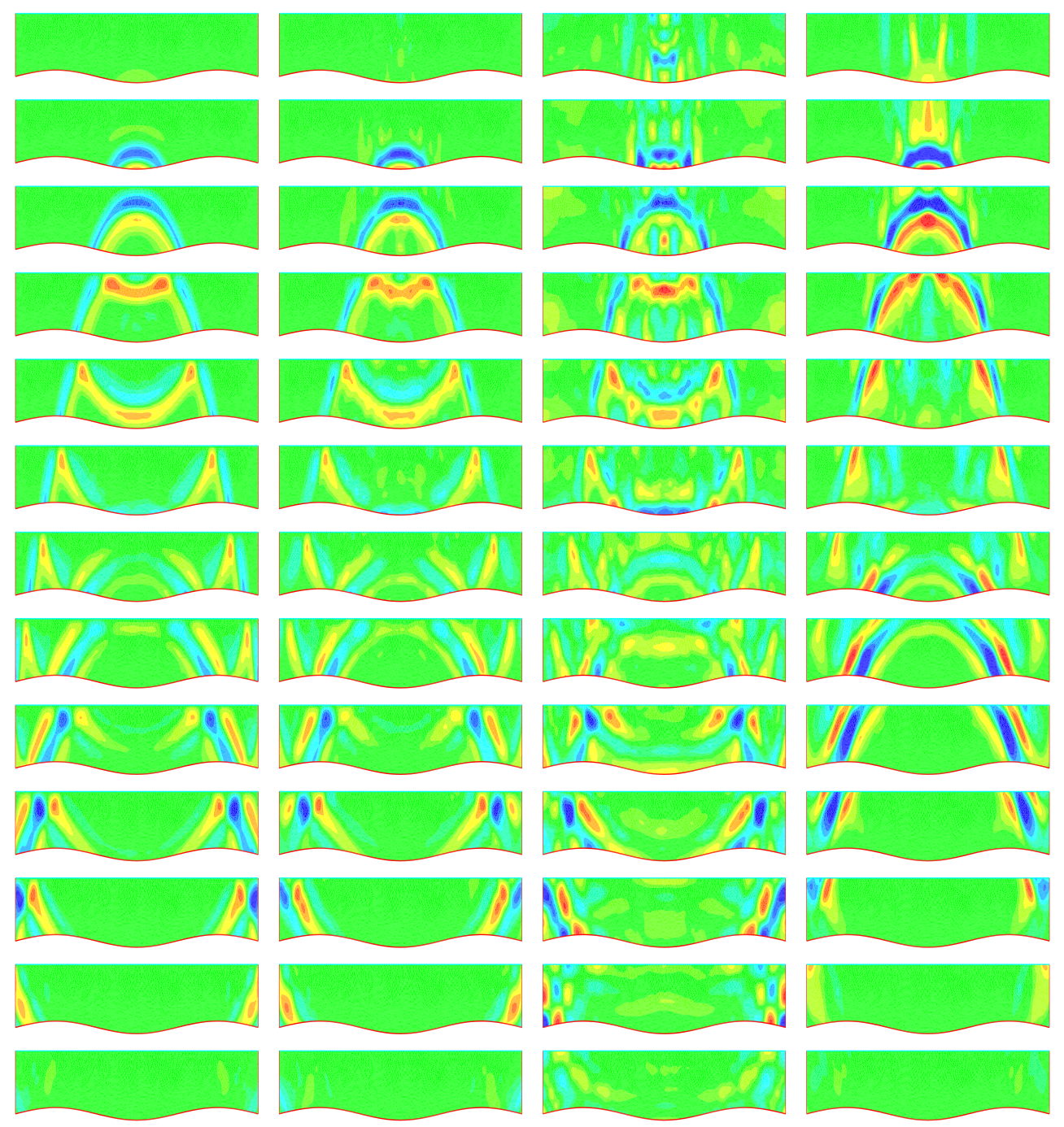

Figure 3: snapshots of the scattered field $p^{S}$ at increasing time steps from top to bottom in the truncated domain $\Omega_{t o p}$ : Column 1: Exact, Column 2: TRAC-ls, Column 3: Neumann-ls and Column 4: TRAC. (without noise in the data) 


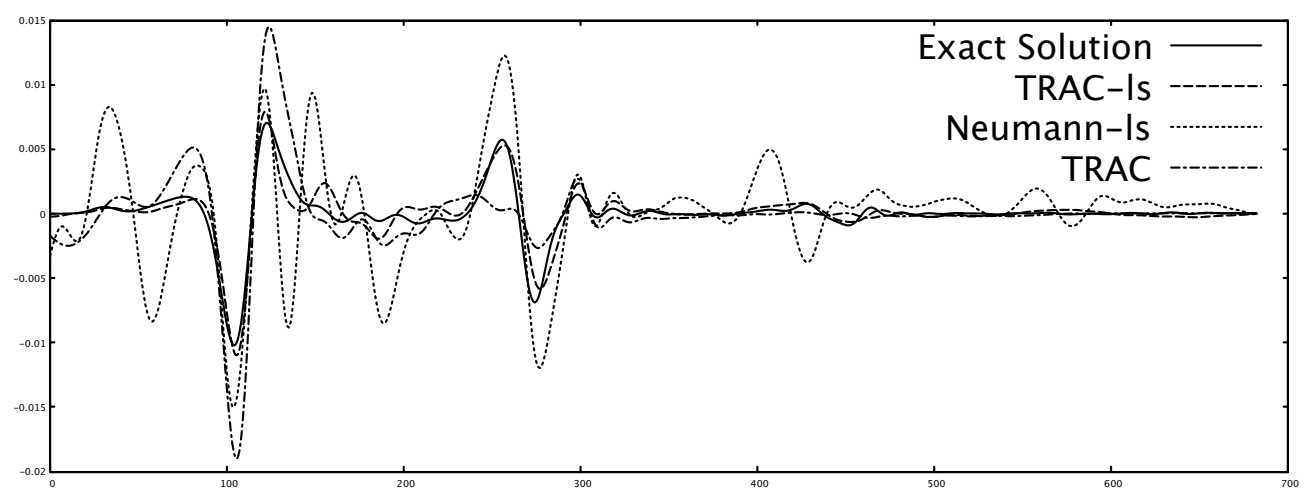

Figure 4: Reconstructed signals $p^{S}$ as a function of time for different methods (without noise in the data)

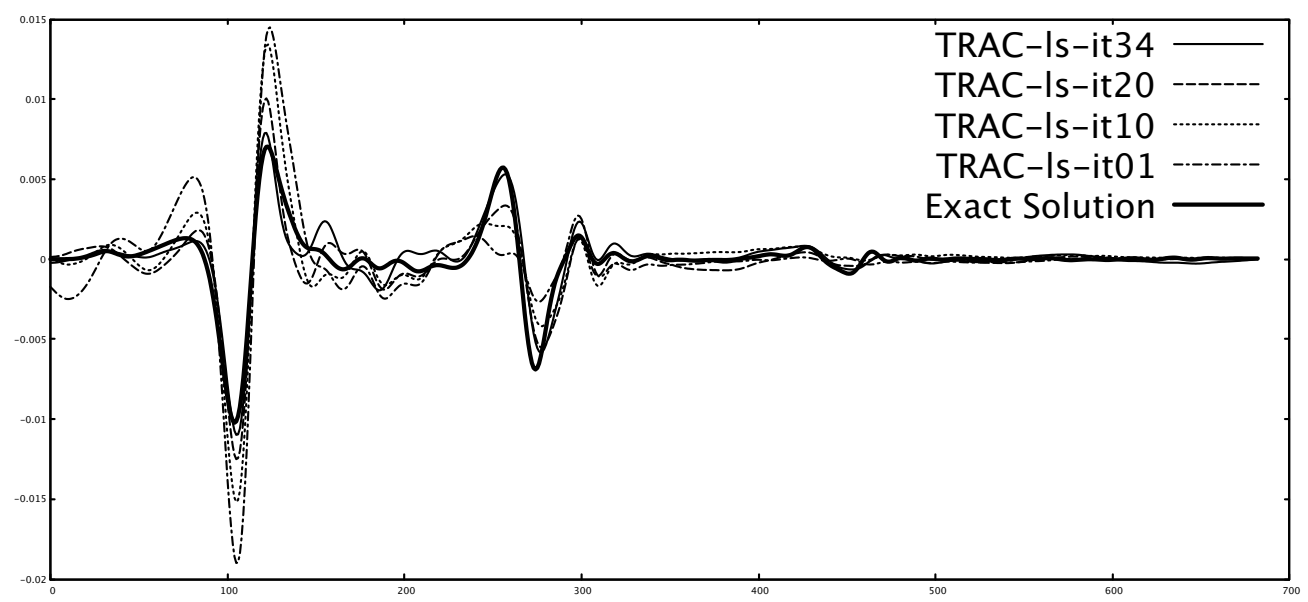

Figure 5: Reconstructed signals $p_{T R A C-l s}^{S}$ as a function of time at various iterations of the conjugate gradient method (without noise in the data) 


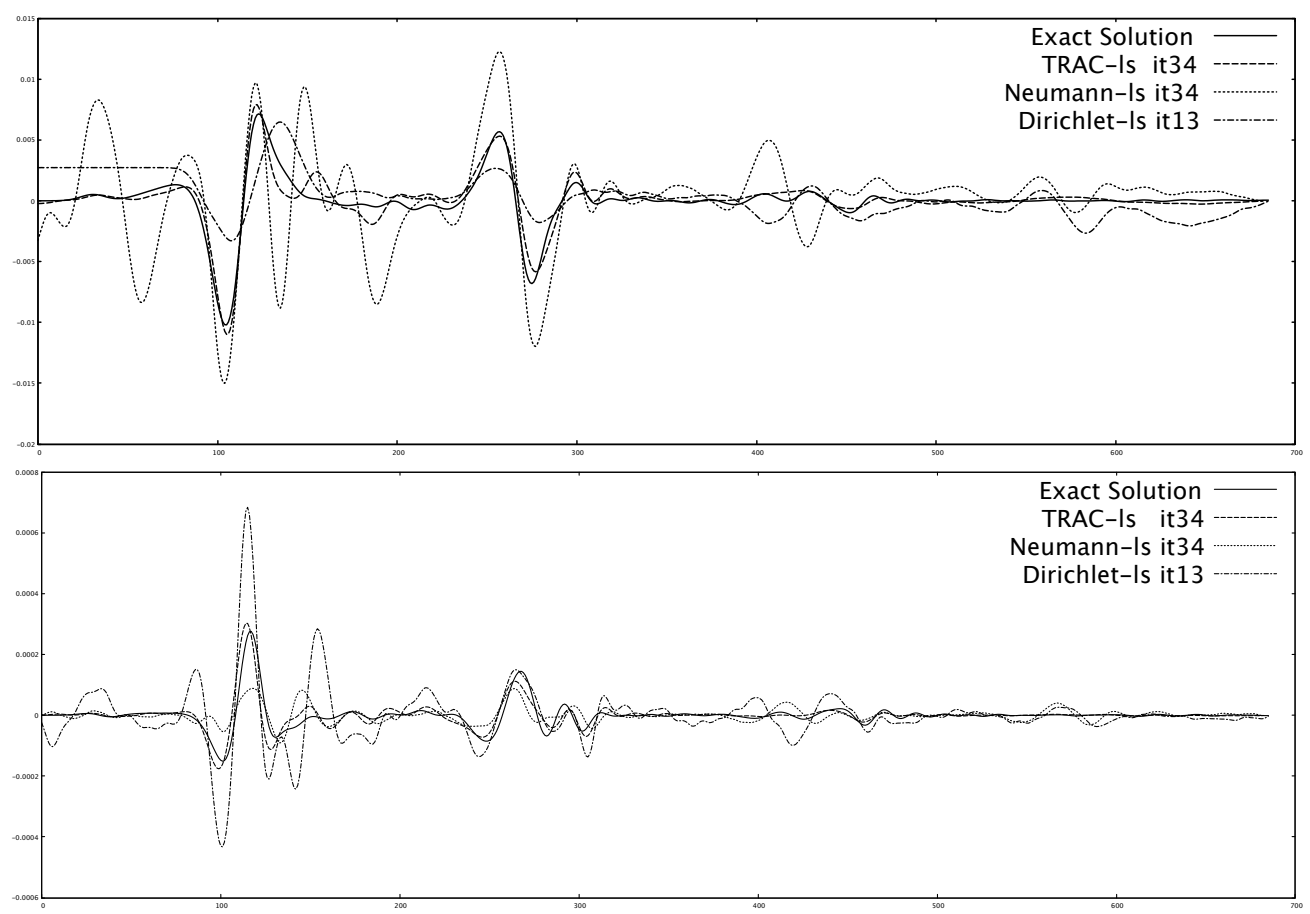

Figure 6: Reconstructed signals $p^{S}$ (top) and $\frac{\partial p^{S}}{\partial n}$ (bottom) as a function of time for various methods (without noise in the data)

middle of the boundary $\Gamma_{\text {redatuming }}$ together with the TRAC-ls, Neumann-ls and Dirichlet-ls. As expected, the TRAC-ls method gives the most accurate results for both quantities. We interestingly note that the Neumann-ls method for the normal derivative gives not so bad results especially compared to its unstable reconstruction of the scattered pressure itself. This may be related to the fact that the Neumann-ls method optimizes the normal derivative on the boundary $\Gamma_{\text {redatuming. }}$ By duality, note that the Dirichlet-ls method for the scattered pressure itself yields quite correct results especially when compared to its unstable reconstruction of the normal derivative. Here also, this may be explained by the fact that the Dirichlet-ls method optimizes the pressure itself

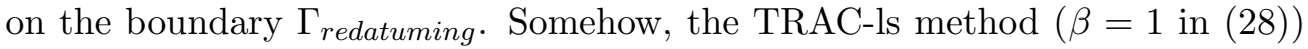
appears as a good choice of the parameter $\beta$ in both cases.

\subsection{Results with noise}

Inverse problems are frequently ill-posed. Hence, a crucial question is the sensitivity of the method with respect to noise in the data. Therefore, we shall add multiplicative Gaussian noise by replacing the recorded data $u_{r e f}^{S}$ on $\Gamma_{S R A}$ by

$$
u_{r e f}^{S}:=(1 .+ \text { Coeff } *(-1 .+2 . * \text { randn })) * u_{r e f}^{S},
$$

where randn satisfies a centered reduced normal law and Coeff is the level of noise taken from $0 \%$ to $20 \%$. 


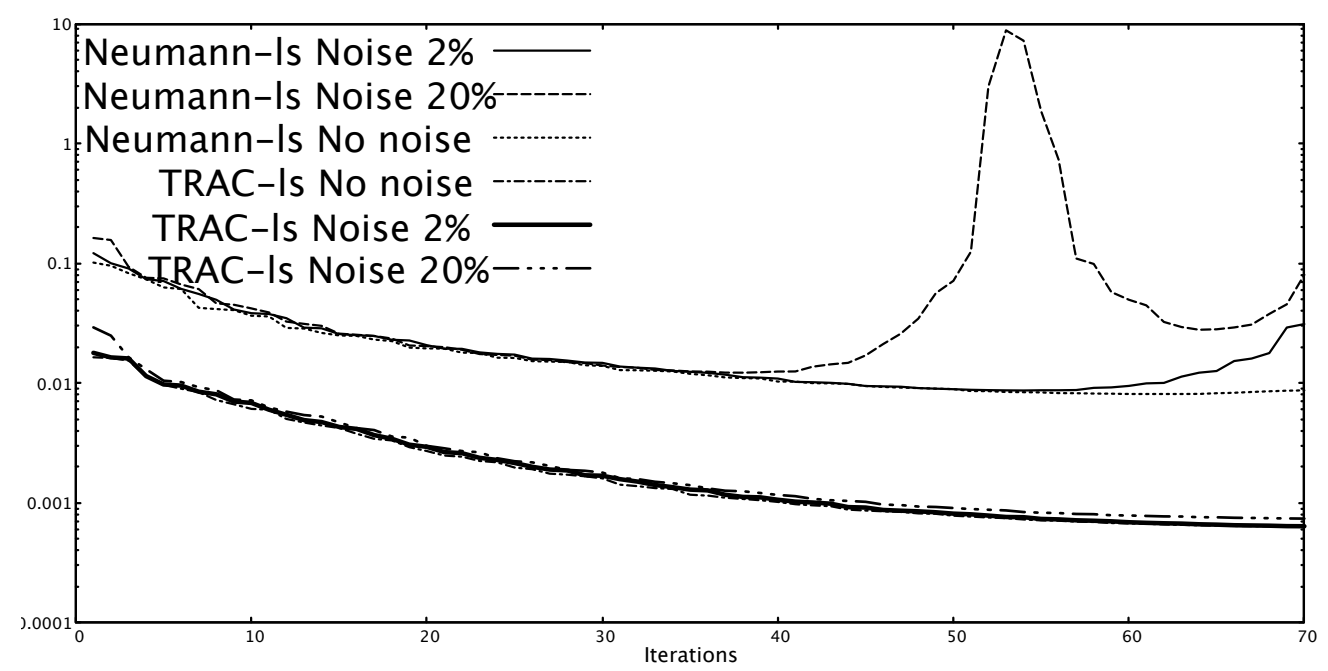

Figure 7: Convergence history of the cost function $(19)$ for the different methods and noise levels

As it is well known, a large number of iterations in the least square problem may decrease the quality of the approximate solution. It is the so-called semi-convergence of the iterative algorithm for solving ill-posed problems with noise in the data. Semi-convergence means that, initially, the iteration vectors approach the noiseless solution while continuing iterations lead to the solution contaminated by noise, see the discussion in [18]. In Fig. 7, we plot the convergence history of the cost function $(19)$ minimized by the conjugate gradient method for the Neumann-ls and TRAC-ls methods with different levels of noise: $0 \%, 2 \%$ and $20 \%$. As expected the Neumann-ls method exhibits a semiconvergence behavior: the higher the noise level, the more problematic the behavior is. Interestingly enough, the TRAC-ls method does not suffer from this problem. In this case, the convergence curves for several levels of noise are almost on top of each other. This is true even not only for a low level of noise $(2 \%)$ but also for a high level of noise $(20 \%)$. This could be related to the robustness of the TRAC method with respect to noise in the data, see [1, 10].

Note also that the initial value of the cost function for TRAC-ls method (which corresponds to the TRAC method) is comparable to the converged value for the Neumann-ls method.

For the sake of completness, we provide Table 2 and Figure 8 which are the counterparts in the noisy case of Table 1 and Figure 4 (noiseless case). For both methods, we show the results obtained after 34 iterations, i.e. just before the start of noise contamination of the Neumann-ls method at a noise level of $20 \%$. Thanks to early stopping for the Neumann-ls method, results without and with noise are comparable.

\subsection{Many Layers}

In this section we consider a more complex geometry where compared to the previous case the regions below and above the redatuming boundary $\Gamma_{\text {redatuming }}$ 


\begin{tabular}{|c|c|c|c|c|}
\hline & Noise level & Neumann-ls & TRAC & TRAC-ls \\
\hline L2-error (global) & $20 \%$ & 93.3 & 120.9 & 39.9 \\
\hline L2-error (redatuming sensors) & $20 \%$ & 31.9 & 25.6 & 13.9 \\
\hline cost function & $20 \%$ & 0.0125 & 0.0287 & 0.0014 \\
\hline
\end{tabular}

Table 2: Errors and cost function value for the different reconstruction methods (20\% noise)

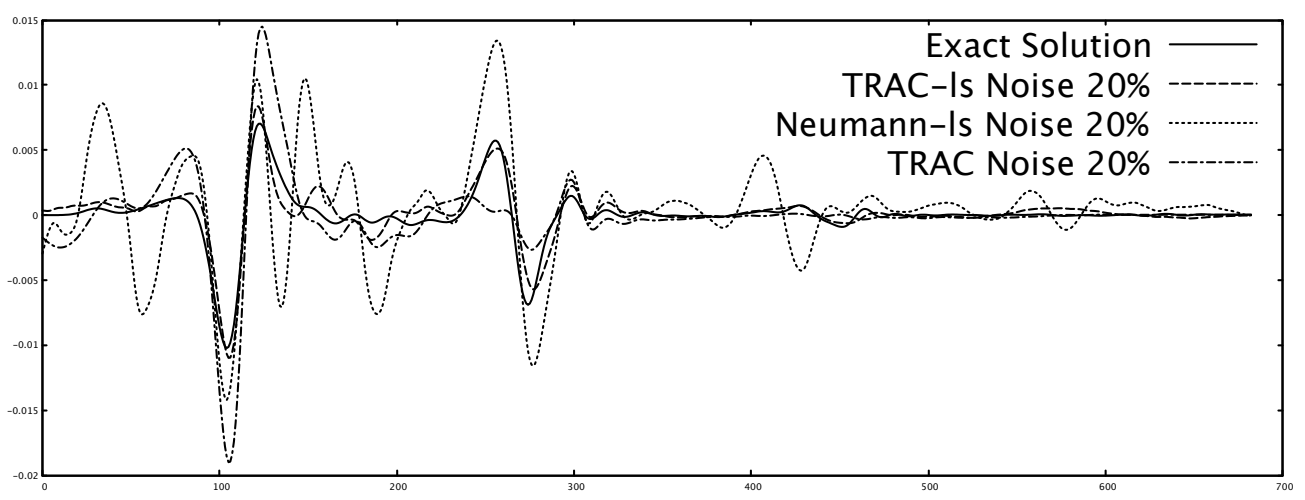

Figure 8: Reconstructed signals $p^{S}$ as a function of time for different methods with $20 \%$ noise level at iteration 34

may be divided into two layers. More precisely in a first experiment we have a three layer domain: two layers below $\Gamma_{\text {redatuming }}$ and still one layer above. In the second experiment, we have a four layer domain by dividing the layer above $\Gamma_{\text {redatuming }}$ into two layers. In addition, we add noise to the velocity field $c$ preserving the arrival times in the following way:

$$
c_{\text {Noise }}=\frac{c}{1+\text { Coeff }_{\text {Noise }} \times \mathcal{N}(0 ., 1 .)}
$$

where $\mathcal{N}(0 ., 1$.$) is a normalized Gaussian noise filtered so that non realistic$ outliers are avoided, Coeff Noise $_{\text {is }}$ the level of noise, see Fig. 9. In this section, we only consider the TRAC-ls method since it gives the best results.

In Table 3, we display the cost function and the L2 error obtained after 69 iterations without noise (lines 1 to 7 ) and with a $10 \%$ noise in the velocities (lines 8 to 14) for seven values of the velocities in the three layers. The first line serves as a reference case and corresponds to a previous numerical illustration since the velocities in layers two and three are the same and there is no noise. The results are very stable with respect to the contrasts in the velocity fields and worsen slightly in presence of noise.

In Table 4, the same data are displayed for a four layer domain without noise or with a $15 \%$ level of noise in the velocities. Here the line 1 serves as a reference test case since it corresponds to the two layer case without noise. As above, this illustrates the robustness of our approach. 


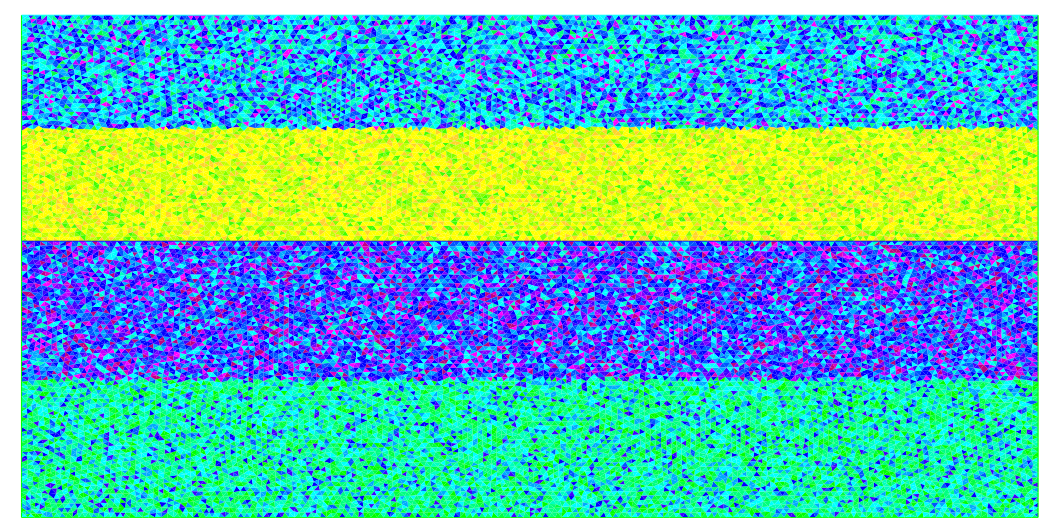

Figure 9: Velocity field with a noise level equals to $15 \%$

\begin{tabular}{|l|c|l|l|}
\hline Noise & Layer Velocities m.s & Cost function & L2-error (global) \\
0.00 & $2800-3200-3200$ & 0.000334427 & 39.9 \\
0.00 & $2800-3200-2800$ & 0.000485026 & 41.8 \\
0.00 & $2800-3200-2400$ & 0.000382373 & 32.9 \\
0.00 & $2800-3200-2000$ & 0.000506179 & 25.3 \\
0.00 & $2800-3200-3600$ & 0.000307094 & 33.8 \\
0.00 & $2800-3200-4000$ & 0.000512521 & 33.5 \\
0.00 & $2800-3200-4400$ & 0.00114314 & 32.8 \\
0.10 & $2800-3200-3200$ & 0.000521904 & 48.1 \\
0.10 & $2800-3200-2800$ & 0.000581837 & 48.8 \\
0.10 & $2800-3200-2400$ & 0.00042535 & 36.5 \\
0.10 & $2800-3200-2000$ & 0.000566084 & 30.5 \\
0.10 & $2800-3200-3600$ & 0.000483933 & 42.5 \\
0.10 & $2800-3200-4000$ & 0.000647048 & 40.4 \\
0.10 & $2800-3200-4400$ & 0.0012518 & 38.4 \\
\hline
\end{tabular}

Table 3: Error and cost function value for different three layer test cases with the TRAC-ls method

\begin{tabular}{|l|l|l|l|}
\hline Noise & Layer Velocities m.s & Cost function & L2-error (global) \\
0.00 & $2800-2800-3200-3200$ & 0.000334427 & 39.9 \\
0.00 & $2800-2000-3200-2800$ & 0.000572001 & 21.6 \\
0.00 & $2800-1200-3200-2400$ & 0.00613234 & 27.8 \\
0.00 & $2800-3600-3200-3600$ & 0.000474359 & 32.8 \\
0.00 & $2800-4400-3200-4000$ & 0.00217664 & 29.5 \\
0.15 & $2800-2800-3200-3200$ & 0.00100535 & 55.1 \\
0.15 & $2800-2000-3200-2800$ & 0.000828798 & 36.9 \\
0.15 & $2800-1200-3200-2400$ & 0.005067 & 32.9 \\
0.15 & $2800-3600-3200-3600$ & 0.00457529 & 58.1 \\
0.15 & $2800-4400-3200-4000$ & 0.0149243 & 46.2 \\
\hline
\end{tabular}

Table 4: Error and cost function value for different four layer test cases with the TRAC-ls method 


\section{Conclusion}

In this paper, we have proposed a redatuming method which is robust with respect to different types of noise. The introduction of an iterative procedure allowed us to generalize the TRAC method to inhomogeneous media. Numerical results demonstrated its robustness with respect to noise even though no regularization was applied. In order to fully assess the relevance of our approach, it should be used in an inverse problem to determine e.g. the physical parameters of an inclusion where as a first step, we would virtually move the sources and receivers closer to the scatterer. Note that when the domain surrounding the inclusion is homogeneous, such an approach was successfully applied in [6].

\section{References}

[1] F Assous, M Kray, F Nataf, and E Turkel. Time-reversed absorbing condition: application to inverse problems. Inverse Problems, 27(6):065003, 2011.

[2] Franck Assous, Marie Kray, and Frédéric Nataf. Time-reversed absorbing conditions in the partial aperture case. Wave Motion, 49(7):617-631, 2012.

[3] Alvin Bayliss and Eli Turkel. Radiation boundary conditions for wave-like equations. Comm. Pure Appl. Math., 33(6):707-725, 1980.

[4] Augustinus Johannes Berkhout. Seismic Migration: Imaging of Acoustic Energy by Wave Field Extrapolation..: Imaging of Acoustic Energy by Wave Field Extrapolation. Elsevier, 2012.

[5] John R Berryhill. Wave-equation datuming. Geophysics, 44(8):1329-1344, 1979.

[6] Maya de Buhan and Marie Kray. A new approach to solve the inverse scattering problem for waves: combining the TRAC and the Adaptive Inversion methods. Inverse Problems, 29(8):085009, 2013.

[7] Bjorn Engquist and Andrew Majda. Absorbing boundary conditions for the numerical simulation of waves. Math. Comp., 31(139):629-651, 1977.

[8] Ioannis Epanomeritakis, Volkan Akçelik, Omar Ghattas, and Jacobo Bielak. A Newton-CG method for large-scale three-dimensional elastic full-waveform seismic inversion. Inverse Problems, 24(3):034015, 2008.

[9] Mathias Fink. Time-Reversal Acoustics. Journal of Physics: Conference Series, 118:012001, 2008.

[10] Dan Givoli and Eli Turkel. Time reversal with partial information for wave refocusing and scatterer identification. Computer Methods in Applied Mechanics and Engineering, 213-216:223 - 242, 2012. 
[11] Marcus J. Grote, Marie Kray, Frédéric Nataf, and Franck Assous. Timedependent wave splitting and source separation. Journal of Computational Physics, 330:981 - 996, 2017.

[12] Eldad Haber, Uri M. Ascher, and Doug Oldenburg. On optimization techniques for solving nonlinear inverse problems. Inverse Problems, 16:1263, 2000 .

[13] F. Hecht. New development in Freefem++. J. Numer. Math., 20(3-4):251$265,2012$.

[14] Michael Hinze, Rene Pinnau, Michael Ulbrich, and Stefan Ulbrich. Optimization with PDE Constraints, volume 23 of Mathematical Modelling: Theory and Applications. Springer Netherlands, first edition, 2009.

[15] Felix Lucka, Nam Huynh, Marta Betcke, Edward Zhang, Paul Beard, Ben Cox, and Simon Arridge. Enhancing compressed sensing 4D photoacoustic tomography by simultaneous motion estimation. SIAM J. Imaging Sciences, 11(4):2224-2253, 2018.

[16] Puyan Mojabi and Joe LoVetri. Microwave Biomedical Imaging Using the Multiplicative Regularized Gauss-Newton Inversion. IEEE Antennas Wireless Propag. Lett., 8:645-648, 2009.

[17] Wim A. Mulder. Rigorous redatuming. Geophysical Journal International, 161(2):401-415, 2005.

[18] Frank Natterer. The mathematics of computerized tomography. SIAM, 2001.

[19] M. Ravasi. An overview of marchenko-based redatuming: past, present, (and future). 2018.

[20] Gerard T Schuster and Min Zhou. A theoretical overview of model-based and correlation-based redatuming methods. Geophysics, 71(4):SI103SI110, 2006.

[21] V Shtivelman and A Canning. Datum correction by wave-equation extrapolation. Geophysics, 53(10):1311-1322, 1988.

[22] Albert Tarantola. Inverse Problem Theory And Methods For Model Parameter Estimation. Society for Industrial and Applied Mathematics, 2005.

[23] Jorn F. M. van Doren, Paul M. J. Van den Hof, Jan D. Jansen, and Okko H. Bosgra. Parameter identification in large-scale models for oil and gas production. In Proc. 18th IFAC World Congress, pages 10857-10862, Milano, Italy, 2011.

[24] Kees Wapenaar, Jan Thorbecke, Joost Van Der Neut, Filippo Broggini, Evert Slob, and Roel Snieder. Marchenko imaging. Geophysics, 79(3):WA39WA57, 2014. 